\title{
Построение концептуальной конфайнмент-модели базы знаний технической диагностики зданий и сооружений
}

\author{
Г.Г.Кашеварова, ПНИПУ, Пермь
}

Своевременная техническая диагностика конструкций зданий и сооружений является важным условием обеспечения их безопасности. Объективность и достоверность информации, предоставляемой экспертами о техническом состоянии строительного объекта, в большой степени зависят от опыта эксперта, то есть от человеческого фактора.

Внедрение интеллектуальных технологий в экспертную деятельность инженера-строителя мотивировано необходимостью передачи знаний опытных экспертов специалистам разной квалификации для расширения и укрепления их профессиональных возможностей. Логика рассуждений человека-эксперта в интеллектуальной системе должна легко поддаваться машинной обработке. Для этого её необходимо структурировать в виде концептуальной схемы системы знаний.

Представлен эффективный метод конфайнмент-моделирования на примере создания концептуальной структуры базы знаний (БЗ) технической диагностики конструкций зданий и сооружений, который позволяет унифицировать процесс разработки онтологических баз знаний, помогает отсеивать неважные факторы и сосредоточиться на тех, которые действительно влияют на результат. Разработанная структура является основой для реализации следующего этапа - построения интегрированной базы знаний интеллектуальной системы диагностики технического состояния строительных объектов разных конструктивных форм и назначения, выполненных из разных материалов, которая должна также включать: методы цифровой обработки результатов лазерного сканирования повреждений конструкций; методы оценки рисков и определения остаточного ресурса конструкций зданий и сооружений с использованием теории нечётких множеств и нечёткой логики; оценку экономического эффекта от мероприятий по обеспечению конструкционной безопасности.

Ключевые слова: техническая диагностика, конструкции, здания и сооружения, интеллектуальные системы, онтология, база знаний, конфайнмент-моделирование.

Conceptual Confinement-Model of a Knowledge Base for Technical Diagnostics of Buildings and Structures

G.G.Kashevarova, PNRPU, Perm

An important condition for ensuring the safety of buildings and structures is timely technical diagnostics. The objectivity and reliability of the information provided by expert on the technical condition of the construction site to a large extent depends on the experience of the expert, i.e. from the human factor.

The introduction of intelligent technologies in the expert activity of a civil engineer is motivated by the need to transfer the knowledge of experienced experts to specialists of different qualifications to expand and strengthen their professional capabilities. The logic of the reasoning of a human expert in an intellectual system should easily yield to machine processing, it must be structured, i.e. present in the form of a conceptual diagram of the organization of the knowledge system.

An effective confinement-modeling method is presented on the example of creating a conceptual structure of the knowledge base for diagnosing the technical condition of buildings and structures, which allows to unify the process of developing ontological knowledge bases, helps to filter out unimportant factors and focus on those that affect the result. The developed structure is the basis for the implementation of the next stage - the construction of an integrated knowledge base of the intellectual system for diagnosing the technical condition of construction objects various structural forms and purposes, made of different materials. This knowledge base should also include: methods for digitally processing the results of laser scanning of structural damage; methods for assessing risks and determining the residual life of buildings structures of using the theory of fuzzy sets and fuzzy logic; economic effect of measures to ensure the safety of building structures.

Keywords: technical diagnostics, structures, buildings and facilities, intelligent systems, ontology, knowledge base, confinement-modeling.

\section{Актуальность темы}

В современном обществе активно развиваются новые технологии, динамично изменяются способы передачи и обработки информации. Достижения в области информационных технологий делают жизнь людей крайне рациональной и стремительной. Виртуальный мир стал неотъемлемой частью жизни, и одна из актуальных задач современности - повышение эффективности использования накопленного ранее теоретического и практического опыта за счёт применения интеллектуальных технологий при решении вновь возникающих проблем.

Это соответствует ключевым положениям Национальной стратегии РФ развития искусственного интеллекта на период до 2030 года для достижения устойчивой конкурентоспособ- 
ности и опережающего развития российской экономики. Реализация этой Стратегии обеспечивается согласованными действиями и приоритетной поддержкой научных исследований российских технологий искусственного интеллекта органами государственной власти и государственными академиями наук (Указ Президента от 10 октября 2019 года № 490, пп. 29, 52).

Модели и методы искусственного интеллекта имеют большие перспективы практического применения в промышленном и гражданском строительстве. Строительные объекты - это социо-технические системы, так как проектируют их, возводят и эксплуатируют люди, которые, как известно, склонны совершать ошибки. В нормах по расчёту и конструированию зданий (сооружений) ошибки людей не учитываются, а требования к безопасной эксплуатации строительных объектов контролируются в РФ на государственном уровне ${ }^{1}$. Своевременная техническая диагностика конструкций зданий и сооружений является важным условием обеспечения их безопасности и направлена на решение двух основных вопросов: как не допустить аварийное разрушение здания или сооружения и как минимизировать негативные последствия, связанные с возможным аварийным разрушением конструкций?

Для достоверного прогноза риска аварии или оценки остаточного ресурса объекта строительства применения лишь классического аппарата теорий прочности и вероятности недостаточно. Основная задача интеллектуальных технологий сегодня - это обработка знаний, связанных с решением сложных вопросов, в которых логическая (или смысловая) информация преобладает над вычислительной [1; 2]. Эта технология позволяет дать строгое математическое описание расплывчатых утверждений эксперта за счёт попытки преодолеть лингвистический барьер между человеком, суждения и оценки которого являются приближёнными и нечёткими, и компьютером, который может выполнять только чёткие инструкции. Техническая диагностика, оценка проектов, принятие решений и прогнозирование, проектирование зданий и сооружений, оптимизация проектных решений - это далеко неполный список перспективных направлений строительной отрасли, где хорошо работают эти технологии.

К сожалению, до сих пор немало специалистов-практиков и учёных скептически относятся к возможностям интеллектуальных технологий. Они сомневаются в том, что эта теория может содействовать решению практических задач. Так обстоит дело с любой новой теорией. Во всяком случае, чтобы судить о теории, надо прежде всего её знать.

\section{Перспективы интеллектуальных технологий для строительной отрасли}

Искусственный интеллект (ИИ) - активно развивающаяся междисциплинарная наука, в которой новые прикладные

${ }^{1}$ Российская Федерация. Законы. Технический регламент о безопасности зданий и сооружений : Федеральный закон от 30 декабря 2009 г. № 384-Ф3. области осваиваются ежедневно. Методы ИИ разнообразны. Они активно заимствуются из других наук, адаптируются и изменяются под решаемые задачи. Можно сказать, что искусственный интеллект - это одновременно и наука, и искусство, и техника, и психология. Это научное направление возникло в 50-х годах XX века на стыке кибернетики, лингвистики, психологии и программирования. Предметом изучения ИИ являются процессы человеческого мышления. Цель - создание модели человеческого интеллекта и реализация её на компьютере. Изначально название этой науки произошло от не совсем удачного перевода основополагающего термина «Artificial intelligence», введённого американским информатиком Джоном Маккарти в 1956 году. Точный перевод слова «intelligence» - «умение рассуждать разумно», а не интеллект (англ. «intellect»). Д. Маккарти, вводя этот термин, пояснял: «Мы понимаем некоторые механизмы интеллекта и не понимаем остальные. Поэтому под интеллектом в пределах этой науки понимается только вычислительная составляющая способности достигать целей в мире».

Знания - это сочетание теоретического понимания проблемы и эмпирических правил (эвристик) для её решения. Источниками знаний, которые хранятся на естественном языке, является обширная база практических и теоретических исследований, представленная в научной и технической литературе, нормативная документация, а также эвристические знания и рассуждения опытных экспертов.

Значительный вклад в появление и развитие теории исскуственного интеллекта внесли Д. Маккарти, Н. Нильсон, Л. Заде, Ф. Хейес-Рот, Д. Уотерман, Д. Ленат, Р. Шенк, Д.А. Поспелов, Э.В. Попов, А.Н. Аверкин, Р.А. Алиев, В.Л. Стефанюк, В.Ф. Хорошевский, Г.С. Осипов, Т.А. Гаврилова, А.П. Ротштейн, М. Минский, Г.В. Рыбина и др. [3-9].

Внедрение интеллектуальных технологий в экспертную деятельность инженера-строителя, связанную с диагностикой, обследованием конструкций зданий и сооружений, мотивировано необходимостью совершенствования технологии принятия решений, касающихся обеспечения безопасности строительных объектов, а также для оценки рисков или определения их остаточного ресурса на основе выявленных при обследовании дефектов несущих, ограждающих конструкций и оснований; для обоснованного принятия решения о реконструкции, капитальном ремонте или сносе здания или сооружения.

Сложная структура строительных конструкций, многофакторность, неполная, иногда недостоверная и противоречивая информация, полученная по результатам инженерного обследования объекта, отсутствие связи между некоторыми нормативными документами, недостаточность сформулированных критериев оценки, проблема «длины шкалы технических состояний» (шкала ГОСТ, включающая всего четыре категории) и размытость границ между ними требуют глубоких знаний в области строительства и в большой степени зависят от опыта эксперта [10]. 
Вопросами технической диагностики эксплуатируемых зданий и сооружений занимались и занимаются многие отечественные, зарубежные учёные и специалисты строительного комплекса А.Г. Ройтман, К.И. Ерёмин, В.В. Леденев, В.Т. Гроздов, В.И. Скрылев, В.Г. Козачек, Н.В. Нечаев, С.Н. Нотенко, И.С. Гучкин, А.И. Бедов, В.В. Знаменский, А.И. Габитов, В.Ф. Сапрыкин, М.Е. Дементьева, А.И. Мальганов, В.С. Плевков, А.И. Полищук, А.Н. Добромыслов, В.В. Габрусенко, В.М. Калинин, С.Д. Сокова, О.С. Вершинина, Н.В. Прядко, В.Г. Казачек и др.

Существует определённый разрыв между техническими разработками методов общего и детального технического обследования строительных конструкций зданий и сооружений [11-14] и методологией организации экспертных знаний для интеллектуальных систем [15-17], а следовательно, и возможностями их более широкого практического применения в строительстве. Это связано с явной нехваткой учебников и специальной литературы, ограниченным финансированием исследований этого чрезвычайно перспективного направления и слабым отечественным рынком программных продуктов для их разработки.

Наиболее распространённый вид прикладных интеллектуальных систем - это экспертные системы (ЭС) [18], использующие профессиональные знания конкретной предметной области. ЭС ориентированы на тиражирование знаний и опыта высококвалифицированных специалистов для решения различных типов задач (диагностики, управления, интерпретации, планирования, конструирования, контроля и др.).

Современное состояние разработок ЭС в строительной отрасли можно характеризовать как стадию всё возрастающего интереса, о чём свидетельствуют как зарубежные, так и отечественные публикации: E. Bernat, L. Gil, K.M. Hamdia Van Balen, H. Li, С.Д. Штовбы, 0.Д. Панкевича, В.А. Соколова, Т.Н. Солдатенко, [19-23] и др.

Внедрение интеллектуальных технологий в экспертную деятельность инженера-строителя способно предоставлять специалистам разной квалификации возможность выбора. Для этой цели в процесс диагностики технического состояния целесообразно ввести дополнительный технологический уровень, включающий использование компьютерных средств обработки знаний (рис. 1).

При этом знания, закладываемые в компьютерно-ориентированную базу знаний (Б3), должны быть организованы и формализованы в соответствии с определёнными структурными правилами, чтобы компьютер мог автономно использовать их при решении конкретной задачи и выдавать результат решения пользователю на основе логического вывода.

Важная задача разработчиков ЭС - продвижение их к конечному пользователю и эффективное функционирование на самом важном этапе «жизненного цикла» - при решении реальных задач.

\section{Основные положения представления структуры базы знаний}

Система ИИ в определённом смысле моделирует интеллектуальную деятельность человека и, в частности, логику его рассуждений. Любая интеллектуальная деятельность человека является по своей сути системной, предусматривающей использование совокупности взаимосвязанных процедур на пути от постановки задачи и целей - к нахождению и использованию решений. А поскольку логика рассуждений человека-эксперта в интеллектуальной системе должна легко поддаваться машинной обработке, её необходимо структурировать, то есть представить в виде концептуальной схемы систему знаний предметной области.

А для этого необходимо:

- иметь профессиональные знания (теоретические и практические);

- понимать процесс мышления человека - опытного эксперта или лица, принимающего решения в конкретном виде деятельности, - иметь возможность выделить основные шаги этого процесса;

- разработать или использовать готовое программное средство, воспроизводящее эти действия.

Для структурирования информации были предложены и опробованы (с переменным успехом) многочисленные модели и методы представления знаний: различные логические модели, семантические и нейронные сети, фреймы, продукционные правила и др. [8; 9; 24].

В последние годы за счет использования новых способов обработки и представления информации на стыке системного анализа, искусственного интеллекта и прикладного анализа данных развивались работы над содержательными онтологиями - концептуальными схемами организации системы знаний конкретных предметных областей и способах целевого использования этих знаний [25-28]. Онтологии широко используются во всех областях, занимающихся обработкой информации на естественном языке.
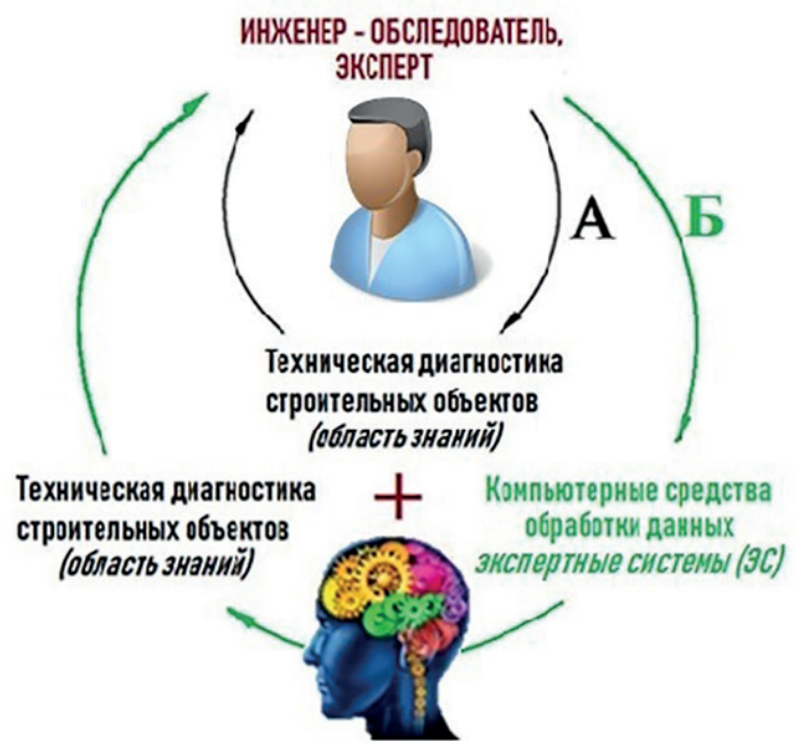

Рис. 1. Концептуальный технологический уровень технической диагностики 
Термин «онтология» в теории искусственного интеллекта используется специалистами как один из основных формализмов представления знаний. Это знания, формально представленные в виде описания множества объектов, понятий и связей между ними.

В простейшем случае построение онтологии сводится к:

- выделению базовых понятий (концептов) данной предметной области;

- построению связей между концептами - определению соотношений и взаимодействий базовых понятий.

Важной особенностью онтологических моделей в интеллектуальных системах является то, что они предназначены не столько для работы с ними людей, сколько для их обработки программами-агентами, а поскольку компьютер не может понимать положение вещей в мире, как человек, ему необходимо представление всей информации в формальном виде.

Компьютерная онтология - формальное выражение концептуальных знаний о предметной области в виде иерархической структуры, которая должна легко поддаваться машинной обработке и анализу, обеспечивая их унифицированное и многократное использование на разных компьютерных платформах, разными исследовательскими группами, при решении разных задач. Для этого все понятия должны быть увязаны, а закономерности установлены, то есть необходимо выявить структуру (каркас) умозаключений эксперта.

Онтологии предметной области обычно строятся экспертами области знания или при их содействии. При этом важны не столько сами понятия, сколько знания людей о данных понятиях и использование их людьми. Реконструируя рассуждения эксперта, чаще всего опираются на две наиболее популярные теории мышления - логическую и ассоциативную.

Традиционная логика формирует критерии, которые гарантируют точность, непротиворечивость общих понятий рассуждений и выводов (классификацию, обобщение, сравнение, категоризацию, умозаключение, абстрагирование, и т.д.). Но человек редко мыслит в категориях математической логики [8], мышление его представляет цепочку идей, связанных общими понятиями. Основными операциями такого мышления являются: ассоциации, приобретённые на основе различных связей; использование прошлого опыта; пробы и ошибки со случайными успехами и пр. Все зависит от конкретных задач и исходного материала, от того, насколько сложна исследуемая предметная область.

Одним из преимуществ использования онтологий в качестве инструмента познания является системный подход к изучению предметной области, который обеспечивает целостный взгляд на предметную область, позволяет восстанавливать недостающие логические связи, делать умозаключения из представленной информации и манипулировать ими. Онтологические модели рассматриваются как базы знаний специального вида.
Поскольку любая модель - это всегда упрощение реальности, то ей свойственна фрагментарность. Но выявляя различные фрагменты знаний, нельзя забывать о главном, то есть важно выделить так называемый «центр ситуации», некий целостный образ или структуру как базис для понимания процессов и явлений, относительно которого развивается знание о предметной области.

Принцип целостности восприятия, как основы мышления, называют гештальтом (нем. Gestalt). Этот термин, заимствованный из гештальт-психологии, ввёл в обиход инженерии знаний Кристиан фон Эренфельс. Самое важное свойство гештальта - стремление к завершению. Это означает, что, выявляя различные фрагменты знаний, нужно помнить о главном, о гештальте, который влияет на остальные компоненты и связывает их в некоторую структуру [8].

При решении реальных задач важно знать, как выделить главное. Метод определения главных компонентов системы и изучения их влияния друг на друга был предложен Т.В. Гагиным [29]. Эту модель принципиально стабильной, самовоспроизводящейся системы

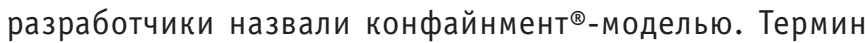
«конфайнмент» (от англ. confinement) взят из физики и дословно обозначает «удержание». Сущность данного подхода заключается в наличии факта замыкания системы в виде системной петли, которая содержит необходимые и достаточные прямые и обратные причинно-следственные взаимосвязи. Элементы системной петли и есть наши фокусы внимания, прицелы - куда нужно смотреть и где искать (рис. 2). При этом считается, что результат в общем случае известен и модель ориентирована на стратегию достижения результата.

Конфайнмент-моделирование - это прикладной инструмент, который позволяет облегчить выстраивание системных моделей и принимать верные решения. В последнее время данный инструмент стал активно применяться в научных разработках различных отраслей [30; 31]. Конфайнментсистема позволяет увидеть ключевые точки и важные взаимосвязи, помогает выделить самое главное и необходимое для решения конкретной задачи. Чтобы понять принципы работы этого инструмента, полезно сопоставить предложенную модель с реальностью.

\section{Пример построения структуры концептуальной модели диагностики технического состояния зданий и сооружений}

Рассмотрим на примере (табл. 1) процесс построения структуры - концептуальной модели интегрированной интеллектуальной системы диагностики технического состояния зданий и сооружений (онтограф) с использованием технологии конфайнмент-моделирования и готового шаблона (см. рис. 2). Этот пример позволит понять правила построения конфайнмент-модели предметной области (рис. 3), увидеть ключевые точки и важные взаимосвязи. 
Главным критерием в принятии решения о степени аварийности или о необходимости проведения мероприятий по приведению объекта строительства к дальнейшей безопасной эксплуатации является категория технического состояния (КТС) здания или сооружения (нормативное, работоспособное, ограниченно работоспособное или аварийное техническое состояние).

Техническая диагностика строительных объектов осуществляется путём сочетания взаимосогласующихся и взаимодополняющих практических, расчётных, исследовательских и аналитических процедур.

Строгое заполнение конфайнмент-модели на уровне здравого смысла (номера элементов на рисунке 2 таблицы 1 соответствуют порядку их определения) позволяет обнаружить не только необходимые для понимания системы элементы, но и их важнейшие взаимосвязи: каждый элемент на своём месте должен быть логично увязан с шестью другими: три должны вызывать его, и три - из него следовать. Таким образом, каждый элемент является одновременно и следствием, и причиной.

Результат построения структуры концептуальной конфайнмент-модели диагностики технического состояния зданий и сооружений представлен в виде онтографа на рисунке 3 таблицы 1, который можно считать онтологией первого уровня, включающей необходимое число системных процессов. Полученная конфайнмент-модель может быть продолжена в обе стороны, то есть быть связующим звеном для других онтологий, построение которых можно свести к аналогичным действиям. При этом на каждом уровне построения конкретной онтологической модели возможно применение разных техник. И примеры такого применения представлены в публикациях [24; 32].

Понимание принципов построения конфайнмент-модели позволяет унифицировать процесс разработки онтологических баз знаний и других предметных областей, облегчить выстраивание структуры в сравнении с известными методами построения онтологий $[8 ; 26 ; 27]$; помогает отсеять неважные факторы (которые казались важными) и сосредоточиться на тех, которые действительно влияют на результат.

Разработанная структура может также являться основой для реализации следующего этапа - построения базы знаний интегрированной интеллектуальной системы технической диагностики строительных объектов разных конструктивных форм и назначения, выполненных из разных материалов, которая также может включать методы цифровой обработки результатов лазерного сканирования повреждений конструкций, методы оценки рисков и определения остаточного ресурса конструкций зданий и сооружений с использованием теории нечётких множеств и нечёткой логики, а также оценку экономического эффекта от мероприятий по обеспечению конструкционной безопасности.

Но при этом следует понимать, что не существует единственно правильного способа моделирования предметной области - всегда есть жизнеспособные альтернативы.
Таблица 1. Процесс построения структуры концептуальной модели

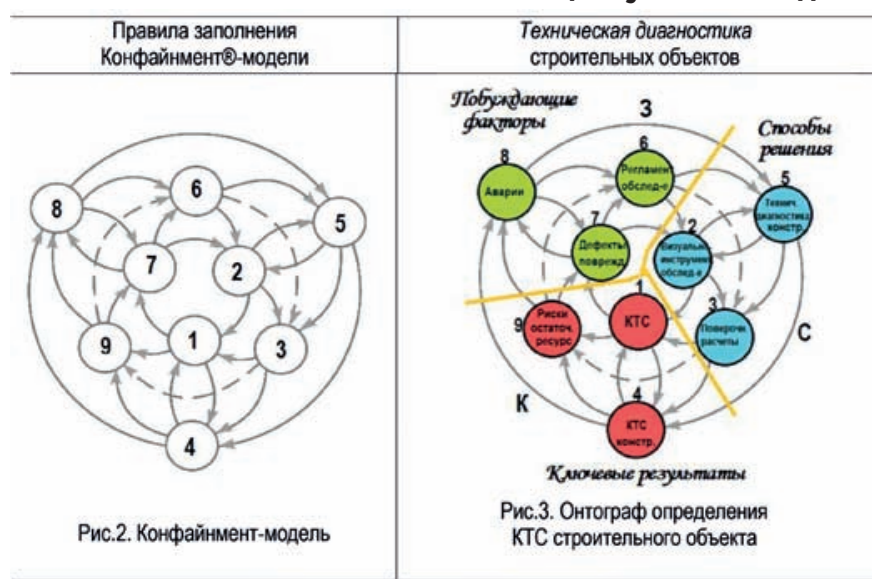

Очередность заполнения (1 проверка)

1 - это собстено результат (иель) си $\begin{array}{ll}1 \text { - это собственно результат (цель) си- } & \begin{array}{l}\text { Lель системы - определить КТ объекта в целом } \\ \text { стемы, её наблюдаемые проявления }\end{array} \\ \text { (1) для оценки рисков разрушения и остаточного ре- }\end{array}$ сурса (9), позволяющих таюке оценить экономический эффект от мероприятий по обеспечению конструкционной безопасности

2,3 и 4-непосредственные причины этого КТС объекта в целом (1) зависит от результатов результата (1). Их выбор диктуется инженерного (визуально-инструментального) следующим требованием. Все три вместе обследования отдельных конструкций (2); они долины с необходимостью и доста- результатов поверочных расчетов - при наличии и точностыо вызывать результат. При этом с учётом выявленных дефектов и повреждений (3); причина (2) долина помимо самого ре- а такке от экспертных оценок КТС отдельных зупьтата вызывать и усиливать причину конструкций здания (4)

(3), а та, в свою очередь, причину (4). При этом элемент (4) должен и сам вызывать результат и одновременно усиливаться им

5 - теперь надо найти такой элемент, Основными задачами технической диагностики кото-рый одновременно вызывает все конструкций зданий и сооружений (5) являются: кото-рый одновременно вызывает все конструкций зданий и сооружений (5) являются: тут важнее, что «все трим. Причина (5) повреждений, обнаруженных в результате инжевзаимосвязана с (2) появления и необходимых расчетов (3), а также от проверки конструкций эксплуата-ционной пригодности и соответствия проектньм пригодности и соответствия проектным
параметрам (4) 6 - это причина для (2) и (5) одновременно

В соответствии с требованием «Правил обследования строительных конструкций зданий и сооружений» и др. нормативных документов инженер-ное обследование (2) и диагностика (5) должны проводится регулярно (6) не реже одного раза в десять лет и не реже одного раза в пять лет для зданий и сооружений или их отдельных элемен-тов, работающих в неблагоприятных условиях

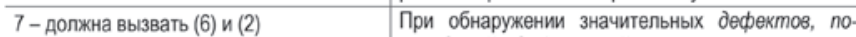
вреждений и деформаций в процессе техни-ческого обслуживания (7) также проводится ин-женерное обследование технического состояния зданий и сооружений $(6,2)$

8 - этот элемент взаимосвязан с (7) и По результатам аварий, последствий пожаров, вызывает (6) и (5) стихийных бедствий, связанных с разрушением стихий инженерное обследование (6) и диагностика (7) 9 - очень важный элемент. Это первая Оценка рисков разрушения и определение серьёзная проверка наших рассуждений. остаточного ресурса (9), являются необходимым Он должен замкнуть систему, то есть следствием при выявленном снижении категории послужить причиной для (7) и (8), будучи технического состояния отдельных констру-ктивных послужить причиной для (7) и (8), будучи
одновременно следствием как причины
элементов (4), а также являются осно-ванием для (4), так и, что важнее, основного принятия решения о ремонте, рекон-струкции или результата системы (1) 1 сносе объекта при обнаружении значительных дефектов, повреждений и дефор-маций (7) или при разрушении зданий в ре-зультате стихийных берствий (8) а также для оцении экономического

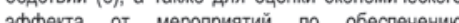
безопасности строительного объекта в целом (1) 
Продолжение таблицы 1

\begin{tabular}{|c|c|}
\hline \multicolumn{2}{|c|}{ Вторая проверка } \\
\hline $\begin{array}{l}\text { Связи, указанные на схеме пунктиром, } \\
\text { представляющие собой замкнутый круг: } \\
\text { «6»- «3»- } 49 \text {, описываются словом } \\
\text { «вызывает» }\end{array}$ & $\begin{array}{l}\text { Проверка выполняется: } \\
\text { замыкание среднего круга: «6» - «3» - «9» для } \\
\text { определения остаточного ресурса и оценки ри-сков } \\
\text { необходимо обследование конструкций, в } \\
\text { соответстви с регламентом, и проведение } \\
\text { расчётов) }\end{array}$ \\
\hline \multicolumn{2}{|c|}{ Третья проверка } \\
\hline $\begin{array}{l}\text { Конфайнмент-модель отчётливо слоится } \\
\text { на три круга: внутренний, средний и } \\
\text { внешний. Внутренний и внешний круги } \\
\text { практически равноправны. А } \\
\text { центральный служит как бы связующим } \\
\text { звеном, пере-давая воздействие из } \\
\text { внутреннего круга во внешний и } \\
\text { наоборот. Средний круг считается } \\
\text { управляющим. } \\
\text { Внутренний и внешний круги в целом } \\
\text { скорее относятся к данностям, к резуль- } \\
\text { татам, а центральный - к промежу- } \\
\text { точным причинам }\end{array}$ & $\begin{array}{l}\text { Проверка практически выполняется. Происходит } \\
\text { передача воздействия из внутреннего круга во } \\
\text { внешний и наоборот }\end{array}$ \\
\hline \multicolumn{2}{|c|}{ Четвертая проверка } \\
\hline $\begin{array}{l}\text { Внутри модели можно выделить три } \\
\text { секто-ра. Отметим их разными цветами: } \\
\text { «Кқ - красный, } 3 \text { - - еелёный, С» - синий. } \\
\text { Элементы в красном секторе описывают } \\
\text { «ключевые результаты», «конкретику». } \\
\text { Это наиболее "реальные», очевидные } \\
\text { части системы. }\end{array}$ & $\begin{array}{l}\text { Элементы группируются в соответствии } \\
\text { принадлежностью К «секторам»: } \\
\text { «К» : 1-4-9- ключевые результаты диагностики; }\end{array}$ \\
\hline $\begin{array}{l}\text { Зелёный сектор является побуждающим } \\
\text { к трансформации конкретики красного в } \\
\text { действия и выводы синего. }\end{array}$ & «3»: 5-2-3 - способы решения задач; \\
\hline $\begin{array}{l}\text { Синий сектор - «процедурно-мыслитель- } \\
\text { ный», охватывает область решений, пра- } \\
\text { вил и выводов. «Синие» элементы - это } \\
\text { «следствия», «смыслы» и «субъективные } \\
\text { суждения» }\end{array}$ & $\begin{array}{l}\text { «Cn: 6-8-7 - побуждающие факторы проведения } \\
\text { диагностики технического состояния. }\end{array}$ \\
\hline $\begin{array}{l}\text { Логика проверок подсказывает } \\
\text { магическую роль элемента, который } \\
\text { одновременно находится } \quad \text { в } \\
\text { «управляющем» центральном круге и } \\
\text { «трансформирующем» зелёном секторе } \\
\text { - элемента (6) в схеме. Он часто } \\
\text { оказывается значимым и решающим }\end{array}$ & $\begin{array}{l}\text { Интересно также отметить попадание } \\
\text { жнеобходимости регулярного обследования } \\
\text { конструкций именно в тот самый (6) элемент, } \\
\text { который является значимым и решающим }\end{array}$ \\
\hline
\end{tabular}

\section{Лuтература}

1. Интеллектуальные информационные системы : Учебное пособие [Электронный ресурс] / Лапина А.В. [и др.]. - Красноярск : СФУ ИКИТ, 2012. - Режим доступа: https://b-ok.cс/ book/2999182/fae901(дата обращения: 27.11.2017).

2. Смагин, А.А. Интеллектуальные информационные системы : учебное пособие / А. А. Смагин, С. В. Липатова, А. С. Мельниченко. - Ульяновск : УлГУ, 2010. - 136 с.

3. Заде, Л.А. Основы нового подхода к анализу сложных систем и процессов принятия решений / Л.А. Заде // Математика сегодня. - М. : Знание, 1974. - С. 5-49.

4. Zadeh, L.A. Fuzzy Logic, Neural Networks, and Soft Computing / L.A. Zadeh // Communications of the ACM. - 1994. - Vol. 37. - № 3. - P. 77-84.

5. Искусственный интеллект : в 3-х кн. Кн.2. Модели и методы : Справочник / Под ред. Д.А. Поспелова - М. : Радио и связь, 1990. - 304 с.

6. Искусственный интеллект : В 3 кн. - М. : Радио и связь, 1990. - Кн. 3: Программные и аппаратные средства / В. Н. Захаров [и др.]; Под ред. В. Н. Захарова, В. Ф. Хорошевского. - М. : Радио и связь, 1990. - 362 с. ISBN 5-256-00366-6
7. Рассел, С. Искусственный интеллект: современный подход / Стюарт Рассел, Питер Норвиг; пер. с англ. и ред. К. А. Птицына; 2-е изд. - М. : Вильямс, 2006. - 1407 с.

8. Гаврилова, Т.А. Базы знаний интеллектуальных систем / Т.А. Гаврилова, В.Ф. Хорошевский. - СПб : Питер, 2000. - 384 с.

9. Ротштейн, А.П. Интеллектуальные технологии идентификации: нечёткая логика, генетические алгоритмы, нейронные сети / А. П. Ротштейн. - Винница: УНИВЕРСУМ, 1999. - 320 с.

10. Классификатор основных видов дефектов в строительстве и промышленности строительных материалов. - Введ. 1993-11-01. - М. : Главгосархстройнадзор России - 95 с.

11. Пособие по практическому выявлению пригодности к восстановлению повреждённых строительных конструкций зданий и сооружений и способам их оперативного усиления. - М. : ЦНИИпромзданий, 1996. - 98 с.

12. Гроздов, В.Т. Признаки аварийного состояния несущих конструкций зданий и сооружений / В.Т. Гроздов. - СПб : Издательский Дом $\mathrm{KN}+$, 2000. - 39 с.

13. Гучкин, И.С. Диагностика повреждений и восстановление эксплуатационных качеств конструкций / И.С. Гучкин. - М. : ACB, 2001. - 171 c.

14. Бойко, М.Д. Диагностика повреждений и методы восстановления эксплуатационных качеств зданий / М.Д. Бойко. - Л. : Стройиздат, 1975. - 336 с.

15. Уэно, Х. Представление и использование знаний / X. Уэно, М. Исидзука. - М : Мир, 1989. - 220 с.

16. Кравченко, Т.К. Современные информационные технологии в развитии компьютерных систем поддержки принятия решений [Электронный ресурс] / Т.К. Кравченко, Н.Л. Наумова. - Режим доступа: http://www.mesi.ru/ksit/k4sem24. zip (дата обращения 01.03.2020).

17. Ручкин, В.Н. Универсальный искусственный интеллект и экспертные системы / В. Н. Ручкин, В. А. Фулин. - СПб : БХВ-Петербург, 2009. - 240 с.

18. Джарратано, Д. Экспертные системы: принципы разработки и программирование / Д. Джарратано, Г. Райлт; пер. с англ. 4-е изд. - М. : 000 «И.Д. Вильямс», 2007. - 1152 с.

19. Van Balen, $K$. Learning from damage of masonry structures, expert systems can help. // III International Seminar on Historical Constructions. November, 2001. - Portugal, Guimaraes, 2001. - P. 15-27.

20. Shtovba, S. Fuzzy rule based system for diagnosis of stone construction cracks of buildings / S. Shtovba, A. Rotshtein, 0 . Pankevich // Advances in Computational intelligence and learning, methods and applications. - Dordrecht : Kluwer Academic Publisher, 2002. - P. 401-412.

21. Соколов, В.А. Оценка технического состояния строительных конструкций зданий на основе многоуровневого вероятностного анализа / В.А. Соколов // Инженерно-строительный журнал. - 2011. - № 7. - С. 45-51. DOI: 10.5862/МСЕ.25.7

22. Солдатенко, Т.Н. Модель идентификации и прогноза дефектов строительной конструкции на основе результатов её обследования / Т.Н. Солдатенко // Инженерно-строительный журнал. - 2011. - № 7 (25). - С. 52-61. 
23. Khader, M. Hamdia. Expert System for Structural Evaluation of Reinforced Concrete Buildings in Gaza Strip Using Fuzzy Logic / Khader M. Hamdia. // A Thesis Submitted in Partial Fulfillment of the Requirement for the Degree of Master of Science in Civil Engineering Rehabilitation and Design of Structure. 2010. 92 p.

24. Рутковская, Д. Нейронные сети, генетические алгоритмы и нечёткие системы / Д. Рутковская, М. Пилиньский, Л. Рутковский ; пер. с польск. И. Д. Рудинского. - М. : Горячая линия-Телеком, 2008. - 452 с.

25. Палагин, А.В. Методика проектирования онтологии Пд0 / А.В. Палагин, Н.Г. Петренко, К.С. Малахов // Комп'ютерні засоби, мережі та системи. - 2011. - № 10. - С. 5-12.

26. Невзорова, О.А. Онтолингвистические системы: методологические основы построения / 0.А. Невзорова // Сборник научных трудов. Том 3. Интеллектуальные системы и технологии. - М. : МИФИ, 2007. - С. 84-85.

27. Клещёв, А.С. Отношения между онтологиями предметных областей. Ч.1. [Электронный ресурс] / А.С. Клещёв, И.Л. Артемьева // Flatik.ru. - Режим доступа: https:/flatik.ru/a-skleshev-i-l-artemeeva-otnosheniya-mejdu-ontologiyami-predm (дата обращения 06.10.2019).

28. Кашеварова, Г.Г. Интеллектуальные технологии в обследовании строительных конструкций / Г.Г. Кашеварова, Ю.Л. Тонков // Academia. Архитектура и строительство. 2018. - № 1. - С. 92-99.

29. Гагин, Т.В. Как выделить главное: принципы Конфайнмент-моделирования [Электронный ресурс]/ Т.В. Гагин, С.С. Бородина. - Режим доступа: http://gagin.tv/index.php?page=28; 2004, http://www.syntone.ru (дата обращения 08.06.2020).

30. Попов, Д.В. Математическое и программное обеспечение конфайнмент-моделирования сложных систем / Д.В. Попов, С.Ю. Поляковский, Н.Н. Мухачева // Принятие решений в условиях неопределённости : межвузовский научный сборник. - 2007. Вып. 4. - Уфа : Уфимск. гос. авиац. техн. ун-т, 2007. - С. 19-26.

31. Мухачева, Н.Н. Системно-когнитивный подход к построению онтологических баз знаний информационноинтеллектуальных ресурсов / Н.Н. Мухачева, Д.В. Попов // Вестник РГРТУ. - 2009. - № 4. - C. 1-8.

32. Kashevarova, G.G. Technical diagnostics of reinforced concrete structures using intelligent systems / G.G. Kashevarova, Y.L. Tonkov Magazine of Civil Engineering. -2020. - № 93 (1). - P. 13-26. ISSN 2071-0305; DOI: 10.18720/MCE.93.2.

\section{References}

1. Authors under the hands Lapina A.V. Intellektual'nye informatsionnye sistemy : Uchebnoe posobie [Intelligent information systems: Textbook]. Access mode: http://files.lib. sfu-kras.ru/ebibl/umkd/228/u_course.pdf (accessed 27.11.2017).

2. Smagin A.A., Lipatova A.A., Mel'nichenko A.S. Intellektual'nye informatsionnye sistemy : uchebnoe posobie [Intelligent information systems: a tutorial]. Ul'yanovsk, UIGU Publ., 2010,136 p.

3. Zade L.A. Osnovy novogo podkhoda k analizu slozhnykh sistem i protsessov prinyatiya reshenii [Foundations of a new approach to the analysis of complex systems and decisionmaking processes]. In: Matematika segodnya [Mathematics today]. Moscow, Znanie Publ., 1974, pp. 5-49.

4. Zadeh L. A. Fuzzy Logic, Neural Networks, and Soft Computing. In: Communications of the ACM, 1994. Vol. 37, no. 3, pp. 77-84. (In Engl.)

5. Iskusstvennyi intellekt [Artificial intelligence] : v 3-kh kn. Kn. 2. Modeli i metody [Models and Methods], Pospelov (ed.). Moscow, Radio and communication Publ., 1990, 304 p.

6. N. Zakharov [et al.]. Iskusstvennyi intellekt [Artificial intelligence] : v 3-kh kn. Kn. 3: Programmnye i apparatnye sredstva [Software and hardware], Pod red. V. N. Zakharov, V. F. Khoroshevskii (eds.). Moscow, Radio and communication Publ., 1990, 362 p. ISBN 5-256-00366-6

7. Rassel, S., Norvig P. Iskusstvennyi intellekt: sovremennyi podkhod [Artificial Intelligence: A Modern Approach], 2-e izd., trans. from English. and ed. K. A. Ptitsyni red. K. A. Ptitsyna. Moscow, Vil'yams, 2006, 1407 p.

8. Gavrilova T.A., Khoroshevskii V.F. Bazy znanii intellektual'nykh sistem [Knowledge base of intelligent systems]. Saint Petersburg, Piter Publ., 2000, 384 p.

9. Rotshtein A.P. Intellektual'nye tekhnologii identifikatsii: nechetkaya logika, geneticheskie algoritmy, neironnye seti [Intelligent identification technologies: fuzzy logic, genetic algorithms, neural networks]. Vinnitsa, Universum Publ., 1999, 320 p.

10. Klassifikator osnovnykh vidov defektov v stroitel'stve $i$ promyshlennosti stroitel'nykh materialov [Classifier of the main types of defects in the construction and building materials industry]. Moscow, Glavgosarkhstroinadzor of Russia Publ., 95 p.

11. Posobie po prakticheskomu vyyavleniyu prigodnosti k vosstanovleniyu povrezhdennykh stroitel'nykh konstruktsii zdanii i sooruzhenii i sposobam ikh operativnogo usileniya [A guide to the practical identification of the suitability for the restoration of damaged building structures of buildings and structures and methods of their operational strengthening]. Moscow, TsNIIpromzdanii Publ., 1996, 98 p.

12. Grozdov V.T. Priznaki avariinogo sostoyaniya nesushchikh konstruktsii zdanii i sooruzhenii [Signs of the emergency state of bearing structures of buildings and structures]. Saint Petersburg, Publishing House KN+ Publ., 2000, 39 p.

13. Guchkin I.S. Diagnostika povrezhdenii i vosstanovlenie ekspluatatsionnykh kachestv konstruktsii [Diagnostics of damage and restoration of operational qualities of structures]. Moscow, ASV Publ., 2001, 171 p.

14. Boiko M.D. Diagnostika povrezhdenii i metody vosstanovleniya ekspluatatsionnykh kachestv zdanii [iagnostics of damages and methods of restoring the operational qualities of buildings]. Leningrad, Stroiizdat Publ., 1975, 336 p.

15. Ueno Kh., Isidzuka M. Predstavlenie i ispol'zovanie znanii [Representation and use of knowledge]. Moscow, Mir Publ., 1989, 220 p.

16. Kravchenko T.K., Naumova N.L. Sovremennye informatsionnye tekhnologii v razvitii komp'yuternykh sistem podderzhki prinyatiya 
reshenii [Modern information technologies in the development of computer decision support systems]. Access mode: http://www. mesi.ru/ksit/k4sem24.zip (accessed 01.03.2020).

17. Ruchkin V.N. Fulin V.A. Universal'nyi iskusstvennyi intellekt $\mathrm{i}$ ekspertnye sistemy [Universal artificial intelligence and expert systems]. Saint Petersburg, BKhV-Peterburg Publ., 2009, 240 p.

18. Dzharratano D. Railt G. Ekspertnye sistemy: printsipy razrabotki i programmirovanie [Expert systems: principles of development and programming], 4-e izd. Moscow, I.D. Vil'yams Publ., 2007, 1152 p.

19. Van Balen, K. Learning from damage of masonry structures, expert systems can help. III International Seminar on Historical Constructions, November, 2001. Portugal, Guimaraes Publ., 2001, pp. 15-27. (In Engl.)

20. Shtovba S., Rotshtein A., Pankevich 0. Fuzzy rule based system for diagnosis of stone construction cracks of buildings [Fuzzy rule based system for diagnosis of stone construction cracks of buildings]. In: Advances in Computational intelligence and learning, methods and applications. Dordrecht, Kluwer Academic Publisher, 2002, pp. 401-412. (In Engl.)

21. Sokolov V.A. Otsenka tekhnicheskogo sostoyaniya stroitel'nykh konstruktsii zdanii na osnove mnogourovnevogo veroyatnostnogo analiza [Assessment of the technical condition of building structures of buildings on the basis of multilevel probabilistic analysis]. In: Inzhenerno-stroitel'nyi zhurnal [Magazine of Civil Engineering], 2011, no. 7, pp. 45-51. D0I: 10.5862/MCE.25.7

22. Soldatenko T.N. Model' identifikatsii i prognoza defektov stroitel'noi konstruktsii na osnove rezul'tatov ee obsledovaniya [Model of identification and forecast of building structure defects based on the results of its examination]. In: Inzhenernostroitel'nyi zhurnal [Magazine of Civil Engineering], 2011, no. 7, pp. 52-61. D0I: 10.5862/MCE.25.8

23. Khader M. Hamdia. Expert System for Structural Evaluation of Reinforced Concrete Buildings in Gaza Strip Using Fuzzy Logic. A Thesis Submitted in Partial Fulfillment of the Requirement for the Degree of Master of Science in Civil Engineering Rehabilitation and Design of Structure, 2010, 92 p.

24. Rutkovskaya, D., Pilin'skii M., Rutkovskii L. Neironnye seti, geneticheskie algoritmy i nechetkie sistemy [Neural networks, genetic algorithms and fuzzy systems]. Moscow, Hotline-Telecom Publ., 2008, 452 p.

25. Palagin A.V., Petrenko N.G., Malakhov K.S. Metodika proektirovaniya ontologii Pd0 [Methods for designing the ontology of PD0]. In: Komp'yuterni zasobi, merezhi ta sistemi [Computer tools, networks and systems], 2011, no. 10, pp. 5-12.

26. Nevzorova 0.A. Ontolingvisticheskie sistemy: metodologicheskie osnovy postroeniya [Ontolinguistic systems: methodological foundations of construction]. In Sbornik nauchnykh trudov. Tom 3. Intellektual'nye sistemy i tekhnologii [Collection of scientific papers. Volume 3. Intelligent systems and technologies]. Moscow, MIFI Publ., 2007, pp. 84-85.

27. Kleshchev A.S., Artem'eva I.L. Otnosheniya mezhdu ontologiyami predmetnykh oblastei. Ch.1. [Relationships between domain ontologies. Part 1]. Website "Flatic.ru". Access mode: https://flatik.ru/a-s-kleshev-i-l-artemeevaotnosheniya-mejdu-ontologiyami-predm (accessed 06.10.2019).

28. Kashevarova G.G., Tonkov Yu.L. Intellektual'nye tekhnologii v obsledovanii stroitel'nykh konstruktsii [Intelligent technologies in the inspection of building structures]. In: Academia. Arkhitektura i stroitel'stvo [Academia. Architecture and construction], 2018, no. 1, pp. 92-99. (In Russ., abstr. in Engl.)

29. Gagin T.V., Borodina S.S. Kak vydelit' glavnoe: printsipy Konfainment-modelirovaniya [How to highlight the main thing: principles of confinement modeling]. Access mode: http:// gagin.tv/index.php?page=28; 2004, http://www.syntone.ru (data obrashcheniya 08.06.2020).

30. Popov D.V., Polyakovskii S.Yu., Mukhacheva N.N. Matematicheskoe i programmnoe obespechenie konfainmentmodelirovaniya slozhnykh sistem / D.V. Popov, S.Yu. Polyakovskii, N.N. Mukhacheva [Mathematical and software for confinement modeling of complex systems]. In: Prinyatie resheniiv v usloviyakh neopredelennosti : mezhvuzovskii nauchnyi sbornik [Decision-making in conditions of uncertainty: interuniversity scientific collection], 2007, Iss. 4. Ufa, Ufa State Aviation Technical University Publ., 2007, pp. 19-26.

31. Mukhacheva N.N., Popov D.V. Sistemno-kognitivnyi podkhod k postroeniyu ontologicheskikh baz znanii informatsionno-intellektual'nykh resursov [System-cognitive approach to the construction of ontological knowledge bases of information and intellectual resources]. In: Vestnik RGRTU [Vestnik of RSREU], 2009, no. 4, pp. 1-8.

32. Kashevarova G.G., Tonkov Y.L. Technical diagnostics of reinforced concrete structures using intelligent systems [Technical diagnostics of reinforced concrete structures using intelligent systems]. In: Inzhenerno-stroitel'nyi zhurnal [Magazine of Civil Engineering], 2020, no. 93 (1), pp. 13-26. ISSN 2071-0305; DOI: 10.18720/MCE.93.2

Кашеварова Галина Геннадьевна (Пермь). Доктор технических наук, профессор, член-корреспондент РААСН. Заведующая кафедрой «Строительные конструкции и вычислительная механика» ФГБОУ В0 «Пермский национальный исследовательский политехнический университет» (614010, Пермь, ул. Куйбышева, 109. ПНИПУ ) Эл. почта: ggkash@mail.ru.

Kashevarova Galina G. (Perm). Professor, Dr.Sc., Corresponding Member of Russian Academy of Architecture and Construction Sciences. Head of department "Building constructions and computational mechanics" Of FGBOU V0 "Perm National Research Polytechnic University; Russia, 614010, Perm, ul. Kuibyshev, 109. PNRPU).E-mail: ggkash@mail.ru. 